\section{Convexity of SMOoth Yield SuRface of Frictional Material}

By Feng-Bao Lin ${ }^{1}$ and Zdeněk P. Bažant, ${ }^{2}$ F. ASCE

\section{INTRODUCTION}

The yield condition, loading surface or inelastic potential of an inelastic material may be described by a surface in the space of principal stresses $\sigma_{1}, \sigma_{2}$ and $\sigma_{3}$. In the deviatoric cross sections, which form equal angles with the principal stress axes, the Tresca criterion is represented by a regular hexagon, which possesses six axes of symmetry, as does the von Mises criterion, which is represented by a circle. For frictional materials, however, only three axes of svmmetry are to be expected, as is exemplified by the Mohr-Coloumb criterion which represents an irregular hexagon. This criterion, however, is not very convenient for numerical finite element analysis because the corners impair convergence of numerical computation. At the same time, material test data are never precise enough to indicate whether the loading surface has comers or is smooth. Therefore, smooth yield conditions should be used in finite element analysis.

Various known smooth yield conditions which have a reduced degree of symmetry in the deviatoric cross section, similar to the Mohr-Coulomb criterion, have been recently reviewed by Zienkiewicz and Pande (1977). Yet, the question of convexity of the yield surface, which is important for theoretical reasons as weil as numerical applications, has apparently, not been examined. This question, will, therefore, be addressed here.

\section{Existing Formulas}

The oldest as well as the simplest formula for a smooth deviatoric cross section which possesses three rather than six axes of symmetry was proposed simultaneously by Gudehus (1973) and by Argyris, et al. (1974). It may be described by the formula

$r(\theta)=r_{c} \frac{2 k}{(1+k)+(1-k) \cos 3 \theta}$

in which $r=$ distance to the yield surface from its axis (i.e., the straight line forming equal angles with the principal stress axes); $k=$ empirica constant and $\theta=$ similarity angle, which is given by the expression cos $3 \theta=J_{3} J_{2}^{-3 / 2} 3 \sqrt{3} / 2$, in which $J_{2}$ and $J_{3}=$ second and third invariants of ${ }^{1}$ Grad. Research Asst., Northwestern Univ., Evanston, IL 60201.

2Prof. of Civ. Engrg. and Dir., Ctr. for Concrete and Geomaterials, Northwestern Univ. - Tech 2410, Evanston, IL 60201

Note.-Discussion open until April 1, 1987. To extend the closing date one month, a written request must be filed with the ASCE Manager of Journals. The manuscript for this paper was submitted for review and possible publication on February 19, 1986. This paper is part of the Joumal of Engineering Mechanics, Vol. 112, No. 11. November, 1986. ASCE. ISSN 0733-9399/86/0011-1259/501.00. Paper No. 21046

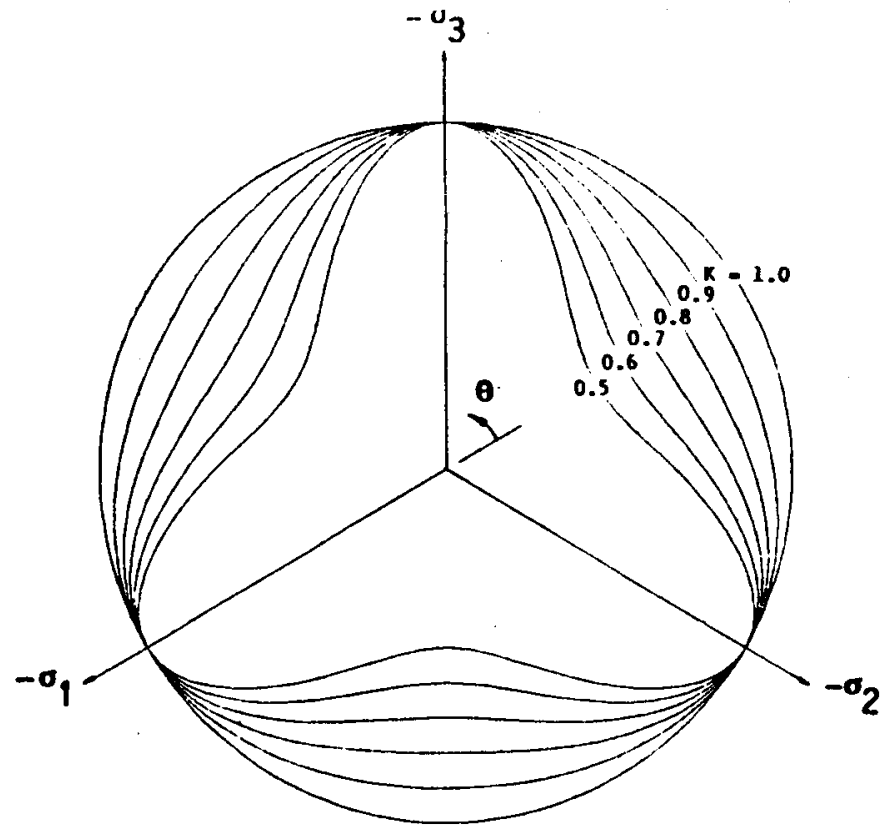

FiG. 1.-Devlatoric Cross Sections According to the Criterion Proposed by Gudehus and by Argyrls, et al.

the stress deviator. Angle $\theta$ is measured from the positive $\sigma_{1}$ direction (see Fig. 1). $r_{c}$ is the value of $r(\theta)$ on the compression meridian, $\theta=60^{\circ}$

Another well known expression was proposed by Willam and Warnke (1975) as part of their five-parameter failure criterion for concrete. This expression has two parameters $r_{c}$ and $r_{t}$ and reads

$r=r_{c} \frac{R+\left(2 r_{t}-r_{c}\right)\left(2 R \cos \theta+5 r_{t}^{2}-4 r_{t} r_{c}\right)^{1 / 2}}{2 R \cos \theta+\left(2 r_{t}-r_{c}\right)^{2}}$

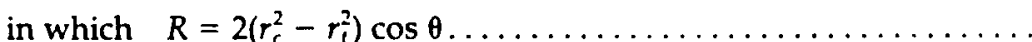

and $r_{t}=$ value of $r(\theta)$ on the tension meridian, $\theta=0^{\circ}$. Eq. 2 represents a section of an ellipse spanning from the tensile direction of one principal stress axis to the compressive stress direction of another principal stress axis; see Fig. 2 (where $k=r_{1} / r_{c}$ ). The ellipse intersects both axes normally, so that the entire deviatoric section, patched up from six identical ellipse segments, has a continuous slope at the axes.

\section{ConvexrTy}

Since the segment of an ellipse is always convex, the yield condition of Willam and Warnke need not be examined. However, the yield condition of Gudehus and Argyris, et al. rreeds to be examined. We do so graphically in Fig. 1, in which the shapes of the deviatoric cross section of this yield criterion are plotted according to Eq. 1. We see from this 


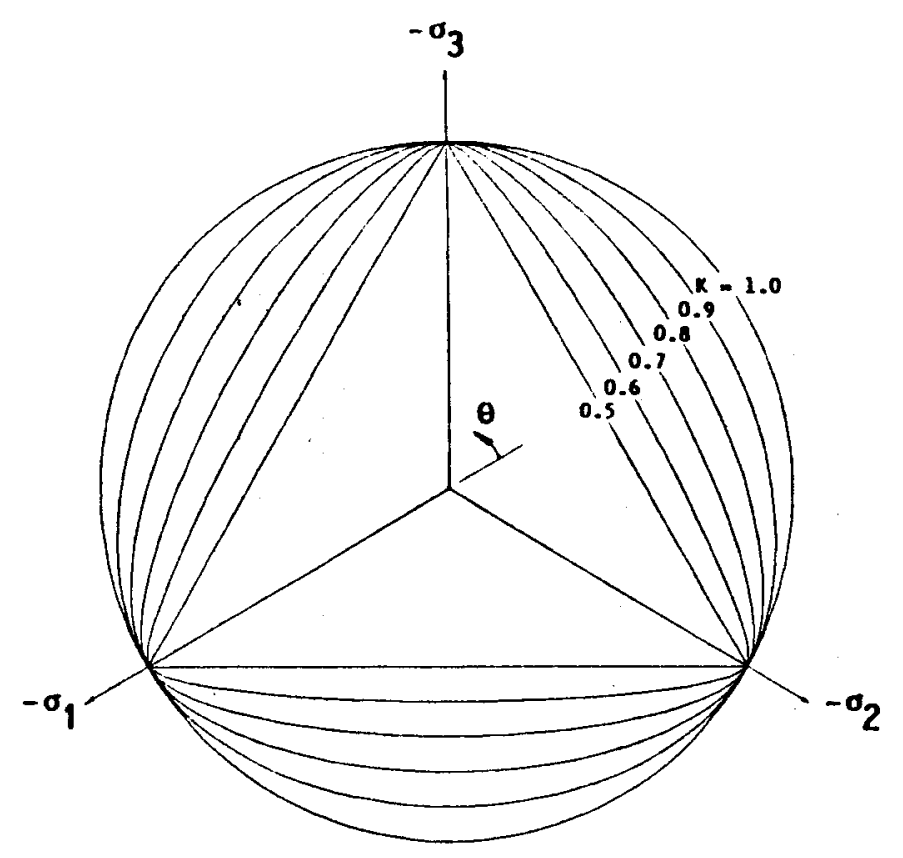

materials, and in such a case the criterion of Willam and Warnke should be preferred despite the fact that it is given by a more complex expression.

\section{APPENDIX.-REFERENCES}

Argyris, J. H., et al. (1974). "Recent developments in the finite element analysis of PCRV." Nuclear Engineering and Design, 28, 42-75.

Gudehus, G. (1973). "Elastoplastische Stoffgleichungen für trockenen Sand." Ingenieur-Archiv, 42, 151-169.

Willam, K. J., and Warnke, E. P. (1975). "Constitutive model for th. triaxial behaviour of concrete." Presented at the Seminar on Concrete Structures Subjected to Triaxial Stresses, ISMES, Bergamo, Italy, 1-30.

Zienkiewicz, O. C. and Pande, G. N. (1977). "Some useful forms of isotropic yield surfaces for soil and rock mechanics." In Finite Elements in Geomechanics, edited by G. Gudehus, John Wiley and Sons, Inc., New York, N.Y. 179-180.

FIG. 2.-Deviatoric Cross Sections According to Willam and Warnke's Criterlon

figure that convexity is violated for small values of $k$. Evaluating the minimum curvature numerically for various values of $k$, and iterating to find the case of zero minimum curvature, we find that the condition of convexity of this criterion is

$k \geq 0.777$

Therefore, if the $k$-values which are less than the foregoing limit cannot be ruled out in advance, the criterion of Gudehus and Argyris, et al. should be replaced by the criterion of Willam and Warnke.

A generalization of the formula of Gudehus and Argyris, et al., which may be written as

$r(\theta)=r_{c} \frac{2 k\left(c_{1}+c_{2} \cos 3 \theta\right)}{\left(c_{3}+k\right)+\left(c_{3}-k\right) \cos 3 \theta}$ with $c_{1}-c_{2}=1, c_{1}+c_{2}=c_{3} \ldots .(5)$

has been also examined. It is found that this type of yield criterion generally does not assure convexity for the entire range of parameter $k$, up to the limiting case of an equilateral triangle (Fig. 2).

\section{Conclusion}

While the yield criterion of Willam and Warnke is always convex, the yield criteria of Gudehus and of Argyris, et al., are convex only for a certain range of material parameters. However, parameter values which fall outside this range might not be excluded for applications to frictional 